\title{
Revisão da Estrutura Fatorial da Escala de Satisfação Conjugal
}

\author{
José Augusto Evangelho Hernandez ${ }^{1}$ \\ Camila Morais Ribeiro \\ Universidade do Estado do Rio de Janeiro, Rio de Janeiro, RJ, Brasil \\ Ana Lúcia Novais Carvalho \\ Universidade Federal Fluminense, Niterói, RJ, Brasil \\ Regina Coeli Tannus Fonseca \\ Faculdade Salesiana Maria Auxiliadora, Macaé, RJ, Brasil \\ Raphael Fischer Peçanha \\ Universidade Estácio de Sá, Rio de Janeiro, RJ, Brasil \\ Eliane Mary de Oliveira Falcone \\ Universidade do Estado do Rio de Janeiro, Rio de Janeiro, RJ, Brasil
}

\begin{abstract}
Resumo
A satisfação conjugal é um construto relevante para o estudo na Psicologia, dada sua importância no desenvolvimento deste relacionamento interpessoal. Esta investigação revisou a estrutura fatorial da Escala de Satisfação Conjugal (ESC). A ESC, frequentemente, tem sido utilizada na pesquisa brasileira, inclusa como medida convergente para gerar evidências de validade de critério no desenvolvimento de novas medidas psicológicas. Por outro lado, suas propriedades psicométricas não são verificadas há 25 anos. Participou deste estudo uma amostra não probabilística de 249 pessoas do Rio de Janeiro, com idades entre 21 e 77 anos $(M=45,3 ; D P=13,26)$, casadas ou em uniões estáveis há 19,5 anos em média $(D P=12,88)$. Os dados coletados foram submetidos às Análises Fatorial Exploratória e Confirmatória. O exame revelou alguns itens com desempenhos psicométricos insatisfatórios. Contudo, uma versão reduzida da ESC apresentou simplicidade e índices de ajuste estimados muito bons. Evidências de validade da existência de um fator de segunda ordem também foram produzidas. A fidedignidade estimada para os fatores e a escala toda variou de 0,93 a 0,85 .
\end{abstract}

Palavras-chave: Satisfação conjugal, relação conjugal, propriedades psicométricas.

\section{Review of the Marital Satisfaction Scale's Factor Structure}

\begin{abstract}
Marital satisfaction is a construct that is relevant to psychology research due to its significance in terms of establishing this interpersonal relationship. The present study examines the factor structure of the Marital Satisfaction Scale (MSS). The MSS has frequently been employed in Brazilian studies, incorporated as a convergent measure for producing criterion validity evidence in the elaboration of new
\end{abstract}

1 Endereço para correspondência: José Augusto Evangelho Hernandez. Rua São Francisco Xavier, 524, $10^{\circ}$ andar, sala 10028D, Maracanã, Rio de Janeiro, RJ, Brasil 20550-900. Fone: (21) 972528440. E-mail: hernandez.uerj@gmail.com 
psychometric methods. Nonetheless, its psychometric properties have not been substantiated for 25 years. A non-probabilistic sample of 249 people from Rio de Janeiro participated in the present study, their ages ranging between 21 and 77 years $(M=45.3 ; S D=13.26)$. They had been married or involved in a civil union (also known as a "domestic partnership" or "common-law marriage" depending on the location) for an average of 19.5 years $(S D=12.88)$. The collected data was assessed via exploratory and confirmatory factor analyses. Such assessment revealed several items exhibiting poor psychometric performance. Nevertheless, an abridged version of the MSS provided simplicity and produced excellent fit indices. It also yielded validity evidence of the existence of a second-order factor. The estimated reliability for both the factors and the entire scale ranged between 0.85 and 0.93 .

Keywords: Marital satisfaction, marital relationship, psychometric properties.

\section{Revisión de la Estructura Factorial de la Escala de Satisfacción Marital}

\section{Resumen}

La satisfacción marital es un constructo relevante para el estudio de la psicología, dada su importancia en el desarrollo de esta relación íntima. Esta investigación examinó la estructura factorial de la Escala de Satisfacción Marital (MSS). El MSS menudo se ha utilizado en la investigación brasileña, incluso como medida para generar criterio de validez convergente en el desarrollo de nuevas medidas psicológicas. Por otro lado, las propiedades psicométricas no se examinan hace 25 años. Participaron en este estudio una muestra no probabilística de 249 personas de Río de Janeiro, 21-77 años de edad ( $M=45.3, D E=$ 13.26), casadas o en unión estable con 19,5 años de media $(D E=12.88)$. Los datos fueron sometidos a un análisis factorial exploratorio y confirmatorio. El examen reveló algunos itemes con actuaciones psicométricas pobres. Sin embargo, una versión reducida de la MSS presentó sencillez y los índices de ajuste estimados muy buenos. También se produjeron evidencia de la validez de la existencia de un factor de segundo orden. La fiabilidad estimada para los factores y la escala completa variaron desde 0.93 hasta 0.85 .

Palabras clave: Satisfacción marital, relación matrimonial, propriedades psicométricas.

O presente estudo examinou a estrutura fatorial da Escala de Satisfação Conjugal (ESC) de Pick de Weiss e Andrade Palos (1988a) na versão brasileira (Dela Coleta, 1989). Este instrumento tem sido usado na pesquisa psicológica nacional inclusive como medida convergente para conferir evidência de validade de critério a novas medidas criadas. No entanto, desde a adaptação da ESC (há mais de 25 anos) não consta da literatura nenhum relato de exame das condições psicométricas da escala.

A satisfação nas relações conjugais é um tema amplamente relatado pela literatura científica da Psicologia. Os indivíduos satisfeitos no casamento apresentaram-se mais saudáveis em comparação com as pessoas que se encontravam insatisfeitas em seus relacionamentos (Gottman \& Silver, 2000). Uma questão bas- tante evidenciada é a associação entre a satisfação conjugal, a felicidade e o bem-estar pessoal (Machado, 2007; Scorsolini-Comin \& Santos, 2011a, 2012). Por outro lado, as relações conjugais insatisfatórias aparecem relacionadas ao surgimento de enfermidades físicas e mentais (Gottman \& Silver, 2000), ao prejuízo das relações parentais (Benetti, 2006; Bolsoni-Silva \& Marturano, 2010), das familiares em geral e das amizades (Norgren, Souza, Kaslow, Hammerschmidt, \& Sharlin, 2004). As características de personalidade (Solomon \& Jackson, 2014), o sexo (Jackson, Miller, Oka, \& Henry, 2014), a presença de filhos (Hernandez \& Hutz, 2009), a escolaridade (Mihalcea, Iliescu, \& Dincă, 2013) a situação socioeconômica (Ahmadi \& Sadeghi, 2016), o exercício de trabalho remunerado (Minnotte, Minnotte, \& Pedersen, 2013), as experi- 
ências românticas anteriores (Mirecki, Chou, Elliott, \& Schneider, 2013) e a dinâmica e as mudanças decorrentes do tempo (Norgren et al., 2004) podem influir aumentando ou diminuindo a satisfação conjugal do casal, conforme o caso.

Pick de Weiss e Andrade Palos (1988a) consideraram a satisfação conjugal como um conjunto de atitudes frente aos aspectos referentes ao cônjuge e às interações no casamento. Estas autoras justificaram a necessidade da criação de uma medida de satisfação conjugal no México visto que a maioria dos instrumentos existentes forneciam medidas globais de satisfação e, em geral, representavam a cultura anglo-saxônica. Por esses motivos criaram a Escala de Satisfacción Marital (ESM) baseada na cultura hispânica.

No primeiro estudo de desenvolvimento da ESM participaram 330 indivíduos casados, 176 mulheres e 154 homens, com idade média de 36,3 anos, o tempo médio de casamento de 13,9 anos e o número médio de filhos de 3,1 (Pick de Weiss \& Andrade Palos, 1988a). Após análises das frequências dos escores dos participantes na ESM, foram selecionados 37 itens que submetidos à Análise Componentes Principais (ACP) com rotação oblíqua resultaram em seis fatores com autovalores $>1,0$, que explicaram $58,1 \%$ da variância total. Por critérios conceituais, foram retidos os três primeiros fatores que explicaram $47,8 \%$ da variância total e excluídos 14 itens. Da análise de conteúdo dos 23 itens remanescentes, foram nomeados os fatores:

1. Satisfação com os aspectos emocionais (SAE) do cônjuge, que se refere à satisfação que o indivíduo tem com as reações emocionais de seu parceiro (por exemplo, como expressa seus sentimentos e se comporta quando tem raiva e ansiedade);

2. Satisfação com a interação conjugal (SIC), que se refere à satisfação que o indivíduo tem com a relação que leva com seu companheiro;

3. Satisfação com aspectos estruturais (SAOE), que se refere à satisfação do indivíduo com a forma de organização, estabelecimento e cumprimento de regras por parte do cônjuge (por exemplo, como organiza a sua própria vida e o impacto disso na vida do parceiro).

No segundo estudo (Pick de Weiss \& Andrade Palos, 1988a), participaram 244 indivíduos casados da cidade do México, 139 mulheres e 105 homens, com idade média de 31,5 anos, tempo de casamento de 8,7 anos e número médio de filhos de 1,7. Foram adicionados 11 novos itens aos 23 itens da ESM selecionados anteriormente (não foi esclarecido o motivo dessa adição). Nova ACP com rotação oblíqua foi executada e extraídos sete fatores com autovalores $>1,0$, que explicaram $49,7 \%$ da variância total. Considerando a clareza conceitual, as pesquisadoras retiveram os três primeiros fatores extraídos que explicaram $45,7 \%$ da variância total. Nos resultados não foi apresentada a matriz rotada de cargas fatoriais dos itens, mas 10 itens foram excluídos e a ESM ficou composta de 24 itens distribuídos em três fatores. Os alfas obtidos para os fatores foram 0,90 para a SIC (10 itens), 0,81, para a SAE (5 itens) e 0,85, para a SAOE (9 itens).

Foram encontradas relações estatísticas significativas $(p<0,05)$ entre as subescalas da ESM e algumas variáveis sociodemográficas (Pick de Weiss \& Andrade Palos, 1988a, 1988b, 1988c). Os homens apresentaram escores médios $>$ as mulheres em SIC e em SAOE. As pessoas com menor tempo de relação (um ou dois anos) mostraram níveis de SIC $>$ pessoas com 16 ou mais anos. Em termos gerais, os níveis de SIC, SAE e SAOE foram menores para os indivíduos com três ou mais filhos do que para os tinham um, dois ou não tinham filhos. Quanto maior o nível da escolaridade dos participantes, maiores os escores médios de SIC. A idade dos indivíduos não apresentou relação estatística significativa com nenhuma das três subescalas da ESM.

Por considerar a ESM uma medida de cultura semelhante à do Brasil, Dela Coleta (1989) fez a adaptação brasileira do instrumento, a Escala de Satisfação Conjugal (ESC). Foram analisados os escores na ESC de 206 sujeitos, 90 homens e 117 mulheres, casados há pelo menos cinco anos e com um filho, no mínimo. A idade dos participantes variou de 23 a 65 anos e os níveis socioeconômicos e de escolaridade foram variados. Os respondentes eram residentes em duas 
cidades de portes médio e grande do interior do Brasil. Após procedimentos simples de tradução e validação de conteúdo da versão brasileira, os dados obtidos na ESC foram submetidos à ACP com rotação varimax. Foi extraída uma solução de cinco fatores com autovalores $>1,0$ que foram responsáveis por $57,2 \%$ da explicação da variância total. Os itens que carregaram no quarto e quinto fatores foram alocados na estrutura original de três fatores baseado no conteúdo dos mesmos e no Scree Plot.

Uma nova ACP com rotação varimax para três fatores foi realizada, que explicaram 48,3\% da variância total. Porém, três itens pertencentes à SAOE ( «A forma como meu cônjuge passa seu tempo livre», «O tempo que passamos juntos», e «As regras que meu cônjuge faz para que sejam seguidas em casa») e um item da subescala SAE («A reação de meu cônjuge quando não quero ter relações sexuais») saturaram mais forte na SIC. A autora sugeriu que estudos futuros verificassem a possibilidade de realocação destes itens onde saturaram mais forte e da reformulação da composição de cada uma das três dimensões da ESC. Além disso, as correlações entre os três fatores bem como os coeficientes de consistência interna obtidos para os mesmos levaram a pesquisadora a considerar uma medida geral de satisfação conjugal (Dela Coleta, 1989).

A ACP foi o método de extração usado para o desenvolvimento (Pick de Weiss \& Andrade Palos, 1988a) e adaptação (Dela Coleta, 1989) da ESC. A ACP não é um genuíno método de análise fatorial. No entanto, aparece nos mais populares pacotes estatísticos como o método de extração default, o que talvez contribua em parte para seu uso indiscriminado. A ACP não distingue a variância comum entre os fatores e a variância total e, em certas condições, pode superestimar a variância explicada pelos componentes (Costello \& Osborne, 2005; Ferrando \& Anguiano-Carrasco, 2010). Uma parte significativa dos estudos psicométricos publicados em importantes periódicos da Psicologia ainda utiliza inadequadamente a Análise de Componentes Principais (ACP) para realizar a tarefa exploratória (Conway \& Huffcutt, 2003; Gaskin \& Happell, 2014; Henson \& Roberts, 2006; Izquierdo, Olea, \& Abad, 2014).
A Análise Fatorial Exploratória (AFE) é mais adequada para o desenvolvimento e validação dos testes psicológicos. Esta técnica explora as variáveis latentes ou os fatores comuns que explicam as respostas dos participantes da pesquisa aos itens de uma medida psicológica (Damásio, 2012; Ferrando \& Anguiano-Carrasco, 2010; Izquierdo et al., 2014; Laros, 2005; Lloret-Segura, Ferreres-Traver, Hernández-Baeza, \& Tomás-Marco, 2014).

$\mathrm{Na}$ adaptação brasileira da ESC (Dela Coleta, 1989) foi utilizada uma rotação ortogonal. As recomendações atuais são para o uso de rotações oblíquas independente dos pressupostos teóricos acerca das relações entre os construtos. Essas orientações estão baseadas no fato de que relações ortogonais perfeitas não serão encontradas em Psicologia (Lloret-Segura et al., 2014).

Os métodos clássicos de tomada de decisão para a retenção de fatores (autovalores e scree plot), atualmente, são considerados inadequados (Lorenzo-Seva, Timmerman, \& Kiers, 2011). A Análise Paralela, com consistência, tem apresentado maior precisão na recomendação de retenção de fatores do que os métodos clássicos (Gaskin \& Happell, 2014).

O critério de Dela Coleta (1989) para selecionar os itens para cada fator na matriz fatorial rotada foi que a carga do item fosse $>0,30$. Porém, esse critério considerou apenas as cargas predominantes em detrimento dos valores das saturações concomitantes em outros fatores. Foram observados sete itens com cross loadings. Sem motivos de ordem teórica que justificassem a manutenção desses itens na ESC, os mesmos podem ser excluídos ou reformulados (Hair, Black, Babin, Anderson, \& Tatham, 2009).

Para verificar o estado do uso da ESC na pesquisa brasileira, foi realizada uma revisão bibliográfica com as bases de dados Literatura Latino-Americana e do Caribe em Ciências da Saúde (LILACS) e Scientific Electronic Library Online (SciELO), compreendendo os artigos publicados no período de 1989 a 2015 no cenário nacional. Os termos utilizados para busca foram "satisfação conjugal" e "marital satisfaction". Foram considerados somente os relatos de pesquisa empírica, que efetivamente utilizaram a ESC na investigação. Foram recuperados oito 
artigos (Dela Coleta, 1992; Oliveira, Falcone, \& Ribas, 2009; Ribeiro, Pinho, \& Falcone, 2011; Sardinha, Falcone, \& Ferreira, 2009; Sbicigo \& Lisboa, 2009; Scorsolini-Comin \& Santos, 2011a, 2011b; Villa \& Del Prette, 2013), os quais revelaram que, após a adaptação da ESC (Dela Coleta, 1989), nenhum estudo de verificação das propriedades psicométricas do instrumento foi realizado.

Diante desse estado, o objetivo do presente estudo foi buscar evidências de validade estrutural para os presentes escores da medida recorrendo às técnicas estatísticas recomendadas pelos estudiosos da Psicometria na atualidade (Gaskin \& Happell, 2014; Izquierdo et al., 2014; Lloret-Segura et al., 2014). Como se apresentarão as condições psicométricas da ESC? Este é o problema que esta pesquisa buscou responder.

\section{Método}

\section{Participantes}

Foram analisados os escores na ESC de 249 indivíduos de uma amostra de conveniência, sendo 151 mulheres $(60,6 \%)$ e 98 homens $(39,4 \%)$ do estado do Rio de Janeiro. A idade dos participantes variou de 21 a 77 anos, média de 45,3 e desvio padrão de 13,26 anos. Todos os sujeitos coabitavam com seus parceiros e estavam envolvidos numa relação conjugal, casamento ou união estável. O tempo de duração da relação variou de 1 a 51 anos com média de 19,5 e desvio padrão de 12,88 anos. Do total da amostra, 225 $(90,4 \%)$ declararam estar experimentando o primeiro casamento, enquanto $24(9,6 \%)$ o segundo casamento. $\mathrm{O}$ número de filhos declarado pelos respondentes variou de 0 a 7 , com média de 1,7 e desvio padrão de 1,11. Do total, 44 pessoas $(17,7 \%)$ não tinham filhos, 55 (22,1\%) tinham um filho, 103 (41,4\%) tinham dois filhos, 37 $(14,9 \%)$ tinham três filhos, oito $(3,2 \%)$ tinham quatro filhos, um $(0,4 \%)$ tinha cinco filhos e um $(0,4 \%)$ tinha sete filhos. Do total de participantes, $149(59,8 \%)$ declararam possuir curso superior completo, $33(13,3 \%)$, superior incompleto, 54 (21,7\%), ensino médio completo, $9(3,6 \%)$, médio incompleto, $3(1,2 \%)$, fundamental completo e $1(0,4 \%)$ não forneceu este dado.

\section{Instrumento}

Foi examinada a adaptação brasileira de Dela Coleta (1989) da Escala de Satisfação Conjugal (ESC) criada por Pick de Weiss e Andrade Palos (1988a). A ESC possui uma medida geral de Satisfação Conjugal (SC) composta de três fatores ou dimensões:

1. Satisfação com a Interação Conjugal (SIC) com 10 itens, que se refere à satisfação de um cônjuge com a relação que tem com o parceiro;

2. Satisfação com Aspectos Emocionais (SAE) com cinco itens, que se refere a satisfação que um cônjuge tem com as reações emocionais do parceiro; $\mathrm{e}$,

3. Satisfação com Aspectos Organizacionais e Estruturais (SAOE) com nove itens, que mede a satisfação que um cônjuge expressa com a organização, o estabelecimento e o cumprimento de normas de seu parceiro. Todos os 24 itens foram medidos por meio de uma escala tipo Likert de três pontos, (1) "Eu gostaria que fosse muito diferente", (2) "Eu gostaria que fosse um pouco diferente" e (3) "Eu gosto de como tem sido".

\section{Coleta de Dados}

Este projeto foi aprovado pela Comissão de Ética da Instituição à qual a pesquisa encontra-se vinculada, mediante parecer 096/2011. Os sujeitos convidados a participar da pesquisa foram informados sobre os objetivos da mesma, leram e assinaram um Termo de Consentimento Livre e Esclarecido. Em geral, os dados foram coletados nas residências dos participantes da pesquisa. $\mathrm{O}$ acesso aos sujeitos se deu por indicação ou amostragem snowball. Uma parte dos indivíduos respondeu à ESC na presença dos pesquisadores e a outra parte, preencheu e devolveu o instrumento posteriormente. Em ambos os casos, a escala preenchida foi devolvida diretamente aos pesquisadores.

\section{Análise dos Dados}

A análise da distribuição dos escores da ESC revelou assimetrias de $-1,325$ a 0,110 ) e curtosis de -1,276 a 0,764 e não foram identifi- 
cados valores extremos. O teste de normalidade multivariada de Mardia (1970) foi $=50,12$, c.r. $=14,74$. Como a ESC é respondida por meio de uma escala Likert de três pontos optou-se pelo uso da matriz de correlação policórica (Dominguez Lara, 2014).

Os dados foram analisados no programa Factor 10.3.01 (Lorenzo-Seva \& Ferrando, 2015) por meio de Análise Fatorial Exploratória (AFE) com método Unweighted Least Squares (ULS) e rotação direct oblimin. Para a determinação do número de fatores a extrair foi utilizada a Análise Paralela baseada em Minimum Rank Factor Analysis (Timmerman \& Lorenzo-Seva, 2011).

Para verificar a existência de um suposto fator de segunda ordem (Dela Coleta, 1989), a matriz de cargas fatoriais também foi submetida à Análise Fatorial Hierárquica, a Solução de Schmid e Leiman (SSL, 1957). A SSL que é uma transformação das matrizes de cargas fatoriais obtidas na análise fatorial de primeira ordem forneceu informações complementares revelando a influência dos fatores de primeira ordem e dos fatores de segunda ordem, de forma independente e não sobreposta, sobre as variáveis observadas (Wolff \& Preising, 2005).

O modelo estrutural original da ESC (Dela Coleta, 1989) com 24 itens, três fatores de primeira ordem e uma dimensão de segunda ordem foi também testado por meio de Análise Fatorial Confirmatória (AFC) com Método de Máxima Verossimilhança e replicação booststrap no AMOS 18 (Arbuckle, 2009). Na busca da adequação da estrutura foram usados os índices: de ajuste global do modelo, razão $\chi^{2} / g l$, que conforme Byrne (2010) deve ser $>2,0$ para indicar um bom ajuste; Goodness of Fit Index (GFI) de proporção de covariâncias explicadas entre as variáveis observadas pelo modelo e o Comparative Fit Index (CFI), ambos devem apresentar valores entre 0,90-0,95 para um bom ajuste (Marôco, 2014); o Root Mean Square Error of Approximation (RMSEA), que testa o ajuste entre o modelo testado e um modelo saturado com os mesmos dados, espera-se que o índice seja $<0,08$ para um ajuste aceitável e $<0,05$ para um muito bom ajuste (Marôco, 2014); da parcimônia Akai- ke Information Criterion (AIC) e Consistent Akaike Information Criterion (CAIC), que são usados na comparação e penalizam a complexidade dos modelos, aquele modelo que apresentar os menores valores tem o melhor ajuste (Byrne, 2010).

\section{Resultados}

A AFE com método ULS e rotação direct oblimin revelou uma solução fatorial de cinco fatores com autovalores $>1,0$. Porém, a Análise Paralela baseada em Minimum Rank Factor Analysis (Timmerman \& Lorenzo-Seva, 2011) recomendou a retenção de três fatores. Na matriz fatorial da nova AFE para três fatores foram identificados alguns problemas estruturais não compatíveis com o modelo original (Pick de Weiss \& Andrade Palos, 1988a). Observou-se que o item "O tempo que minha mulher/meu marido dedica ao nosso casamento" apresentou carga fatorial de 0,19 para a dimensão SIC (para a qual foi designado), de -0,04 em SAE e de 0,67, em SAOE. O item da SIC, "O comportamento de minha mulher/meu marido na frente de outras pessoas", apresentou cargas fatoriais de 0,06 em SIC, 0,32 em SAE e 0,30 em SAOE. O item da SIC, "O tempo que dedica a mim", saturou com cargas de 0,33 em SIC, de 0,01 em SAE e 0,59 em SAOE. O item da SAE, "A reação de minha mulher/meu marido quando não quero ter relações sexuais", apresentou cargas de 0,34 em SAE, -0,05 em SAOE e 0,36 em SIC. O item da SAOE, "O tempo que passamos juntos" saturou 0,38 em SAOE, 0,07 em SAE e 0,32 em SIC. O item da SAOE, "A forma como minha mulher/ meu marido procura resolver os problemas.", saturou 0,46 em SAOE, 0,34 em SAE e 0,16 em SIC.

Esses seis itens foram excluídos com base em Hair et al. (2009) e uma nova AFE, mesmo método anterior, foi realizada com os 18 itens restantes. O teste $\mathrm{KMO}$ forneceu um valor de 0,85 (considerado bom) e o Teste de Esfericidade de Bartlett apresentou os valores, $\chi^{2}{ }_{(153)}$ $=1.463,90, p<0,001$, ambos indicando a adequação dos dados à análise fatorial. A matriz de correlação policórica dos itens pode ser vista na 
Tabela 1

Matriz de Correlações Policóricas dos Itens da ESC

\begin{tabular}{|c|c|c|c|c|c|c|c|c|c|c|c|c|c|c|c|c|c|c|}
\hline Itens & 2 & 3 & 4 & 5 & 6 & 8 & 9 & 11 & 12 & 13 & 14 & 15 & 16 & 17 & 19 & 20 & 21 & 24 \\
\hline 2 & 1,00 & & & & & & & & & & & & & & & & & \\
\hline 3 & 0,51 & 1,00 & & & & & & & & & & & & & & & & \\
\hline 4 & 0,73 & 0,39 & 1,00 & & & & & & & & & & & & & & & \\
\hline 5 & 0,54 & 0,32 & 0,39 & 1,00 & & & & & & & & & & & & & & \\
\hline 6 & 0,61 & 0,62 & 0,54 & 0,42 & 1,00 & & & & & & & & & & & & & \\
\hline 8 & 0,37 & 0,39 & 0,55 & 0,23 & 0,45 & 1,00 & & & & & & & & & & & & \\
\hline 9 & 0,35 & 0,34 & 0,29 & 0,22 & 0,35 & 0,18 & 1,00 & & & & & & & & & & & \\
\hline 11 & 0,19 & 0,37 & 0,24 & 0,09 & 0,49 & 0,34 & 0,34 & 1,00 & & & & & & & & & & \\
\hline 12 & 0,30 & 0,42 & 0,26 & 0,20 & 0,44 & 0,34 & 0,40 & 0,80 & 1,00 & & & & & & & & & \\
\hline 13 & 0,26 & 0,34 & 0,22 & 0,25 & 0,40 & 0,23 & 0,30 & 0,73 & 0,72 & 1,00 & & & & & & & & \\
\hline 14 & 0,38 & 0,46 & 0,36 & 0,21 & 0,49 & 0,28 & 0,26 & 0,65 & 0,76 & 0,63 & 1,00 & & & & & & & \\
\hline 15 & 0,31 & 0,24 & 0,06 & 0,31 & 0,21 & 0,15 & 0,32 & 0,13 & 0,17 & 0,15 & 0,20 & 1,00 & & & & & & \\
\hline 16 & 0,36 & 0,40 & 0,24 & 0,28 & 0,47 & 0,29 & 0,39 & 0,26 & 0,23 & 0,19 & 0,20 & 0,60 & 1,00 & & & & & \\
\hline 17 & 0,31 & 0,43 & 0,18 & 0,25 & 0,44 & 0,23 & 0,38 & 0,29 & 0,33 & 0,28 & 0,28 & 0,36 & 0,65 & 1,00 & & & & \\
\hline 19 & 0,24 & 0,27 & 0,08 & 0,25 & 0,20 & 0,08 & 0,11 & 0,20 & 0,22 & 0,14 & 0,20 & 0,38 & 0,26 & 0,19 & 1,00 & & & \\
\hline 20 & 0,24 & 0,08 & 0,21 & 0,16 & 0,24 & 0,06 & 0,46 & 0,21 & 0,23 & 0,19 & 0,33 & 0,26 & 0,30 & 0,28 & 0,23 & 1,00 & & \\
\hline 21 & 0,45 & 0,55 & 0,51 & 0,42 & 0,57 & 0,47 & 0,28 & 0,39 & 0,35 & 0,38 & 0,37 & 0,25 & 0,38 & 0,38 & 0,28 & 0,23 & 1,00 & \\
\hline 24 & 0,35 & 0,41 & 0,22 & 0,13 & 0,30 & 0,26 & 0,30 & 0,36 & 0,37 & 0,31 & 0,37 & 0,42 & 0,42 & 0,39 & 0,20 & 0,27 & 0,47 & 1,00 \\
\hline
\end{tabular}

Tabela 2

Análise Paralela baseada em Minimum Rank Factor Analysis

\begin{tabular}{cccc}
\hline Variáveis & \multicolumn{3}{c}{$\%$ de variância } \\
\cline { 2 - 4 } & Dados Atuais & Média Aleatória & $95^{\circ}$ Percentil Aleatório \\
\hline 1 & $41,5^{* *}$ & 11,5 & 12,8 \\
2 & $11,7^{* *}$ & 10,5 & 11,5 \\
3 & $9,7^{*}$ & 9,6 & 10,6 \\
4 & 6,0 & 8,9 & 9,6 \\
\hline
\end{tabular}

Nota. Número de 500 matrizes aleatórias de correlação policórica. Método permutação de dados brutos (Buja \& Eyuboglu, 1992). ${ }^{* *}$ Número de fatores recomendados quando o $95^{\circ}$ percentil é considerado. ${ }^{*}$ Número de fatores recomendados quando a média é considerada.

Tabela 1. Foram extraídos cinco fatores com autovalores $>1,0$. A Análise Paralela baseada em Minimum Rank Factor Analysis (Timmerman \& Lorenzo-Seva, 2011) manteve a recomendação de retenção de três fatores (Tabela 2).

Todos os 18 itens da versão reduzida saturaram mais fortes nos fatores para os quais esta- vam designados no modelo teórico, sendo que 12 deles apresentaram cargas fatoriais $>0,50$, de razoáveis a excelentes (Hair et al., 2009). O fator SAOE ficou com sete itens, o SIC com sete itens e o SAE com quatro itens. Para todos os fatores e a medida global (ESC) a fidedignidade estimada variou de 0,85 a 0,93 (Tabela 3 ). 
O Índice de Simplicidade de Bentler (1977) foi $0,99\left(100^{\circ}\right.$ percentil $)$ e o Índice de Simplicidade de Cargas (Lorenzo-Seva, 2003) foi 0,52 $\left(100^{\circ}\right.$ percentil). Estes valores indicaram que cada item representa, predominantemente, uma única dimensão e a solução global apresentou um elevado grau de simplicidade. A Root Mean Square of Residuals (RMSR) foi 0,0520, enquanto o valor médio esperado para um modelo aceitável de até 0,0635 , segundo o critério de Kelley (1935).

Tabela 3

Cargas Fatoriais da AFE com Rotação Direct Oblimim dos Itens da ESC e Comunalidades

\begin{tabular}{|c|c|c|c|c|c|}
\hline & Itens & SAOE & SIC & SAE & $h^{2}$ \\
\hline 16 & As prioridades que minha mulher/meu marido tem na vida. & $\mathbf{0 , 8 2}$ & 0,06 & $-0,06$ & 0,69 \\
\hline 15 & A forma como minha mulher/meu marido organiza sua vida e suas coisas. & $\mathbf{0 , 7 7}$ & $-0,08$ & $-0,06$ & 0,52 \\
\hline 17 & A forma como passa o seu tempo. & $\mathbf{0 , 6 0}$ & 0,04 & 0,13 & 0,45 \\
\hline 24 & As regras que minha mulher/meu marido faz para que sejam seguidas em casa. & $\mathbf{0 , 4 4}$ & 0,06 & 0,24 & 0,36 \\
\hline 09 & O tempo que dedica a si mesma (o). & $\mathbf{0 , 3 9}$ & 0,09 & 0,21 & 0,31 \\
\hline 20 & O cuidado que minha mulher/meu marido tem com sua saúde. & $\mathbf{0 , 3 7}$ & 0,01 & 0,15 & 0,19 \\
\hline 19 & A pontualidade de minha mulher/meu marido. & $\mathbf{0 , 3 5}$ & 0,03 & 0,07 & 0,16 \\
\hline 04 & A frequência com que minha mulher/meu marido me abraça. & $-0,15$ & 0,93 & $-0,03$ & 0,76 \\
\hline 02 & A frequência com que minha mulher/meu marido me diz algo bonito. & 0,16 & 0,77 & $-0,06$ & 0,67 \\
\hline 06 & A comunicação com minha mulher/meu marido. & 0,16 & $\mathbf{0 , 5 7}$ & 0,24 & 0,62 \\
\hline 08 & A forma como me pede para termos relações sexuais. & $-0,01$ & $\mathbf{0 , 5 1}$ & 0,14 & 0,34 \\
\hline 21 & O interesse que minha mulher/meu marido tem pelo que eu faço. & 0,29 & $\mathbf{0 , 5 0}$ & 0,17 & 0,51 \\
\hline 05 & A atenção que minha mulher/meu marido tem para com minha aparência. & 0,21 & $\mathbf{0 , 4 7}$ & $-0,07$ & 0,31 \\
\hline 03 & O quanto minha mulher/meu marido me atende. & 0,22 & $\mathbf{0 , 4 3}$ & 0,22 & 0,47 \\
\hline 12 & A forma como se comporta quando está chateada (o). & 0,01 & $-0,02$ & $\mathbf{0 , 9 2}$ & 0,84 \\
\hline 11 & A forma como se comporta quando está triste. & $-0,01$ & $-0,05$ & $\mathbf{0 , 9 1}$ & 0,79 \\
\hline 13 & A forma como se comporta quando está preocupada (o). & $-0,01$ & 0,01 & $\mathbf{0 , 7 9}$ & 0,62 \\
\hline \multirow[t]{5}{*}{14} & A forma como se comporta quando está de mau humor. & $-0,01$ & 0,14 & $\mathbf{0 , 7 3}$ & 0,63 \\
\hline & Variância Explicada & 2,75 & 3,08 & 3,41 & \\
\hline & Proporção da Variância (\%) & 29,8 & 33,3 & 36,9 & \\
\hline & Fidedignidade estimada & 0,84 & 0,89 & 0,93 & \\
\hline & Correlações entre Fatores & & & & \\
\hline $\mathrm{F} 2$ & & 0,42 & & & \\
\hline F3 & & 0,36 & 0,41 & & \\
\hline
\end{tabular}

Nota. Método de extração: Unweighted Least Squares. Em negrito, as cargas fatoriais mais fortes dos itens nos fatores.

Na Tabela 4 (resultado da SSL) podem ser observadas essas relações diretas (cargas fatoriais) entre as variáveis observadas, os fatores de primeira (SAE, SIC e SAOE) e segunda ordem (G1) de forma independente. $\quad \mathrm{Na}$ análise das cargas fatoriais da SSL (Tabela 4) pode ser ob- servado os itens da ESC mostraram saturações suficientes para representar, simultaneamente, suas dimensões específicas (SAE, SIC e SAOE) e a Satisfação Conjugal (G1). Os itens 19 e 20 foram exceções: o primeiro não apresentou saturações suficientes para representar as dimensões 
Tabela 4

Cargas Fatoriais da SSL para Fatores de Primeira e Segunda Ordens da ESC

\begin{tabular}{|c|c|c|c|c|c|}
\hline & Itens & SAOE & $\mathrm{SIC}$ & SAE & G1 \\
\hline 16 & As prioridades que minha mulher/meu marido tem na vida. & 0,66 & 0,04 & $-0,04$ & 0,50 \\
\hline 15 & A forma como minha mulher/meu marido organiza sua vida e suas coisas. & $\mathbf{0 , 6 1}$ & $-0,06$ & $-0,05$ & 0,37 \\
\hline 17 & A forma como passa o seu tempo. & $\mathbf{0 , 4 8}$ & 0,03 & 0,10 & 0,46 \\
\hline 24 & As regras que minha mulher/meu marido faz para que sejam seguidas em casa. & 0,35 & 0,04 & 0,19 & $\mathbf{0 , 4 5}$ \\
\hline 09 & O tempo que dedica a si mesma (o). & 0,31 & 0,06 & 0,17 & 0,42 \\
\hline 20 & O cuidado que minha mulher/meu marido tem com sua saúde. & 0,29 & 0,01 & 0,12 & $\mathbf{0 , 3 1}$ \\
\hline 19 & A pontualidade de minha mulher/meu marido. & 0,28 & 0,02 & 0,06 & 0,28 \\
\hline 04 & A frequência com que minha mulher/meu marido me abraça. & $-0,12$ & 0,67 & $-0,03$ & 0,53 \\
\hline 02 & A frequência com que minha mulher/meu marido me diz algo bonito. & 0,13 & 0,55 & $-0,05$ & $\mathbf{0 , 5 9}$ \\
\hline 06 & A comunicação com minha mulher/meu marido. & 0,13 & 0,41 & 0,19 & 0,63 \\
\hline 08 & A forma como me pede para termos relações sexuais. & $-0,01$ & 0,37 & 0,11 & $\mathbf{0 , 4 3}$ \\
\hline 21 & O interesse que minha mulher/meu marido tem pelo que eu faço. & 0,17 & 0,36 & 0,13 & $\mathbf{0 , 5 7}$ \\
\hline 05 & A atenção que minha mulher/meu marido tem para com minha aparência. & 0,16 & 0,34 & $-0,05$ & $\mathbf{0 , 4 1}$ \\
\hline 03 & O quanto minha mulher/meu marido me atende. & 0,18 & 0,31 & 0,18 & $\mathbf{0 , 5 6}$ \\
\hline 12 & A forma como se comporta quando está chateada (o). & 0,01 & $-0,01$ & 0,74 & 0,54 \\
\hline 11 & A forma como se comporta quando está triste. & $-0,01$ & $-0,04$ & $\mathbf{0 , 7 3}$ & 0,50 \\
\hline 13 & A forma como se comporta quando está preocupada (o). & $-0,01$ & 0,01 & $\mathbf{0 , 6 3}$ & 0,47 \\
\hline 14 & A forma como se comporta quando está de mau humor. & $-0,01$ & 0,10 & $\mathbf{0 , 5 8}$ & 0,53 \\
\hline G1 & $\mathrm{ESC}_{\text {total }}$ & 0,60 & 0,69 & 0,60 & \\
\hline
\end{tabular}

Nota. G1= Dimensão global. Em negrito, as cargas fatoriais mais fortes dos itens nos fatores específicos e global.

específica (SAOE) e geral; o segundo, mostrou carga fatorial abaixo do mínimo aceitável para representar o fator específico SAOE, mas apresentou o suficiente para representar a dimensão geral.

Na busca de confirmação dos resultados obtidos por meio da exploração, os modelos original (24 itens) e reduzido (18 itens) da ESC, ambos com uma dimensão de segunda ordem e três dimensões de primeira ordem, foram testados e comparados pela AFC (Figura 1). Nos resultados, o modelo reduzido em comparação com o modelo original apresentou melhor ajuste considerando os índices estimados (Tabela 5).

O Coeficiente de Correlação de Pearson não apurou correlações estatísticas significativas entre os fatores SIC, SAE, SAOE e a $\mathrm{ESC}_{\text {total }}$ e o Tempo de Duração da Relação ou a Idade dos respondentes. Por outro lado, para os participantes do sexo feminino foram apuradas correlações negativas significativas entre Idade $(r=-0,20$; $p<0,01)$, Tempo de Duração da Relação $(r=$ $-0,16 ; p<0,05)$ e o fator SIC.

O Teste $t$ de Student para amostras independentes $\left(t_{(247)}=2.879, d=0,37\right)$ apurou diferenças estatísticas significativas $(p<0,01)$ entre os escores médios de homens $(2,47)$ e mulheres $(2,29)$ no fator SAOE. Para os fatores SIC, SAE e $\mathrm{ESC}_{\text {total }}$ não foram apuradas diferenças sexuais e os tamanhos dos efeitos calculados foram todos muito pequenos, $d<0,20$ (Cohen, 1992).

Para efeitos de análise, a variável Número de Filhos foi dividida em quatro categorias: sem filho, um filho, dois filhos, três ou mais filhos. A Análise de Variância One-Way para o fator Número de Filhos e as variáveis SIC, SAE, SAOE 

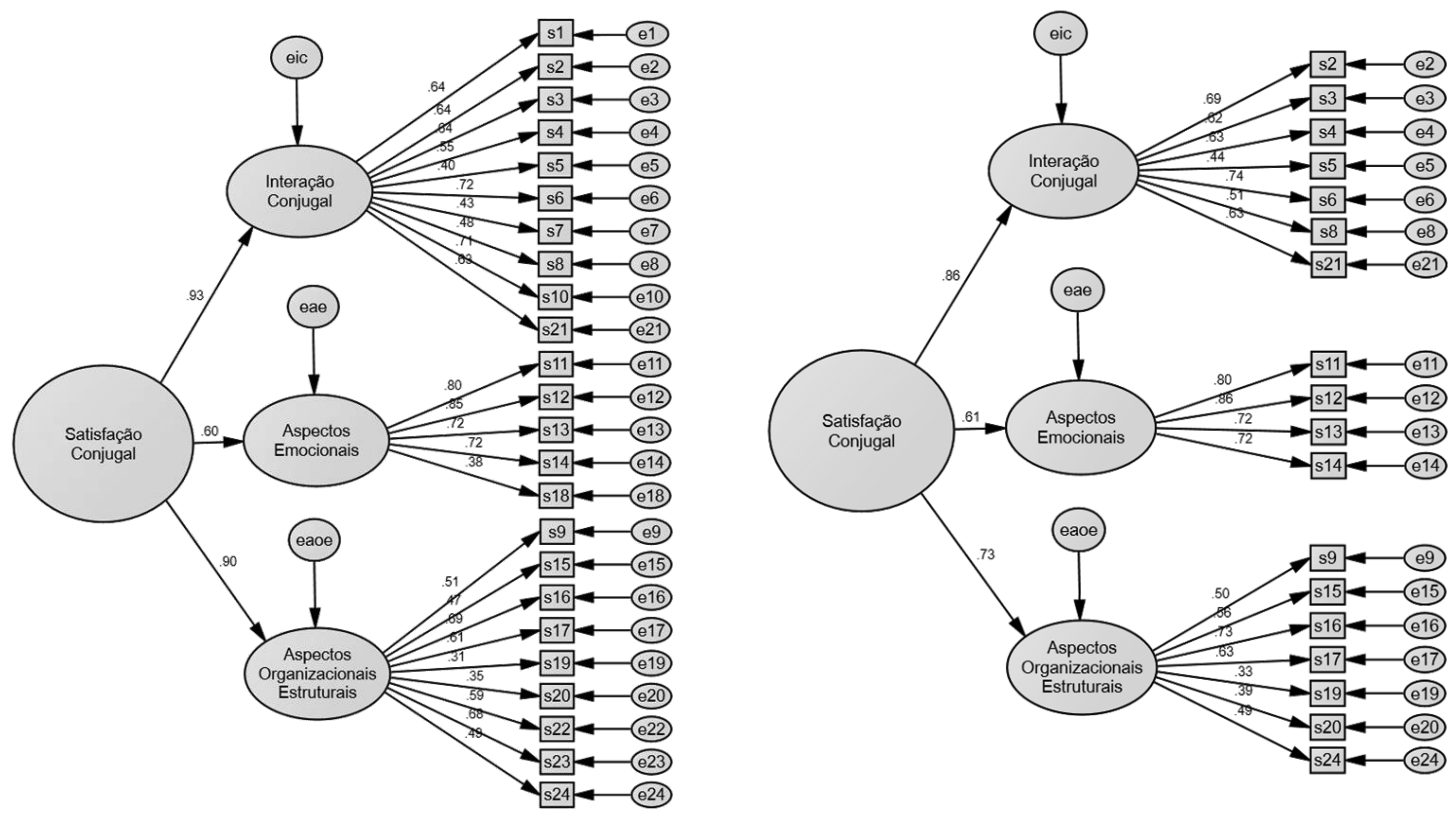

Figura 1. Diagramas dos modelos original e reduzido da ESC comparados com suas estimativas padronizadas, respectivamente.

Tabela 5

Índices de Adequação da AFC para os Modelos Original e Reduzido da ESC

\begin{tabular}{lrrrrrr}
\hline Modelos & $\chi^{2} / D F$ & GFI & CFI & RMSEA (LO90-HI90) & AIC & CAIC \\
\hline Original (24 itens) & 2,267 & 0,841 & 0,844 & $0,071(0,064-0,079)$ & 666,434 & 896,824 \\
Reduzido (18 itens) & 1,921 & 0,901 & 0,910 & $0,061(0,050-0,072)$ & 331,618 & 507,799 \\
\hline
\end{tabular}

e $\mathrm{ESC}_{\text {total }}$ identificou diferenças estatísticas significativas entre os escores médios dos grupos comparados nas variáveis $\mathrm{SIC}, F_{(3,245)}=3.224, p$ $<0,05$, SAOE, $F_{(3,245)}=3.013, p<0,05$, e ESCtotal, $F_{(3,245)}=2.556, p<0,05$. O teste post hoc de Hochberg identificou essas diferenças entre os grupos comparados: (a) Para a SIC e a ESC total' as diferenças significativas foram entre os grupos de participantes sem filhos $(M=2,7 ; D P=$ 0,36 e $M=2,5 ; D P=0,32$, respectivamente) e com apenas um filho $(M=2,4 ; D P=0,50$ e $M=$ 2,$3 ; D P=0,45$, respectivamente). Os indíviduos sem filhos apresentaram mais SIC e ESC $_{\text {total }}$ com o cônjuge do que os que possuiam apenas um filho. Os tamanhos dos efeitos foram $d=0,62$ e $d$ $=0,56$, respectivamente. Não foram identicadas diferenças significativas entre os demais grupos; (b) Para a SAOE, a diferença significativa foi entre o grupo de participantes com três ou mais filhos $(M=2,45 ; D P=0,44)$ e o grupo de apenas um filho $(M=2,20 ; D P=0,52)$. Os indivíduos com três ou mais filhos estavam mais satisfeitos com a forma de organização, estabelecimento e cumprimento de regras por parte do cônjuge do que o grupo de apenas um filho. $\mathrm{O}$ tamanho do efeito foi $d=0,52$. Não foram identificadas diferenças significativas entre os demais grupos.

A maioria dos participantes $(n=182)$ possuíam curso superior completo e incompleto, os outros níveis da Escolaridade apresentaram número reduzido de participantes. Assim, não foi viável examinar as relações entre os níveis da Escolaridade e a Satisfação Conjugal.

\section{Discussão}

No estudo atual, alguns itens da ESC foram excluídos, por apresentarem cargas mais fortes em fatores não designados no modelo original, cross loadings ou cargas fatoriais insuficientes, alguns desses itens coincidiram com os itens de desempenhos psicométricos pobres do estudo de Dela Coleta (1989). Mas, como os métodos utilizados nos dois estudos foram diferentes, fi- 
cou dificultada a comparação entre os mesmos. A AFE atual extraiu uma solução final com 18 itens, sete da SAOE, sete da SIC e quatro da $\mathrm{SAE}$, que gerou evidências de validade fatorial para a ESC e que foi confirmado pela AFC.

Pick de Weiss e Andrade Palos (1988a) não usaram a ESC como uma medida global. Mas Dela Coleta $(1989$, p. 107) defendeu essa possibilidade, contudo sua ideia estava embasada apenas nos "índices de confiabilidade obtidos" em seu estudo. A SSL, usada no estudo atual, é um recurso que pode auxiliar no desenvolvimento do conteúdo dos construtos mais amplos (segunda ordem) ou mais específicos (primeira ordem), representados nos instrumentos de medida. $\mathrm{O}$ conhecimento acerca da contribuição relativa dos diferentes níveis de fatores é de relevância teórica, uma vez que indica a relação entre a especificidade e a generalidade. Na versão reduzida da ESC (Tabela 4) as variáveis observadas representaram bem o construto global salvaguardando os construtos específicos. A AFC também revelou um bom ajuste para o modelo reduzido da ESC com a presença de uma dimensão geral. Concomitante, a AFC revelou um ajuste considerado sofrível (Marôco, 2014) para o modelo original da ESC com 24 itens (Tabela 5).

Assim, na presente pesquisa por meio de procedimentos exploratórios foram geradas evidências de validade fatorial para a ESC reduzida com um fator global e três fatores de primeira ordem. Essa solução fatorial primou pela parcimônia, haja vista o alto grau de simplicidade obtido (Bentler, 1977; Lorenzo-Seva, 2003). Além disso, evidenciou um bom ajuste $(\mathrm{GFI}=0,99) \mathrm{e}$ os resíduos apresentaram-se abaixo do esperado (Kelley, 1935). A fidedignidade estimada para o modelo revelou valores muito bons para todos os fatores e escala geral. Além disso, esse modelo reduzido da ESC foi submetido a AFC e apresentou um bom ajuste (Tabela 5).

Nesta pesquisa os homens apresentaram mais SIC do que as mulheres, contudo o tamanho desse efeito foi de moderado a pequeno (Cohen, 1992). Pick de Weiss e Andrade Palos (1988a, 1988b) e Rostami, Ghazinour, Nygren e Richter (2014) também encontraram homens mais satisfeitos do que mulheres.
Uma das explicações dessas diferenças está baseada nos diferentes papéis de mulheres e homens no casamento. Os papéis femininos seriam mais exigentes e menos gratificantes do que os papéis masculinos. As mulheres teriam uma percepção menos positiva dos seus casamentos e sentir-se-iam menos beneficiadas. As mulheres casadas com emprego em tempo integral se deparariam com mais responsabilidades, deveres e conflitos de papéis dentro de suas famílias (Rostami et al., 2014). Outra explicação refere que as mulheres teriam expectativas maiores por intimidade e apoio emocional e os homens não seriam socializados para fornecer este tipo de apoio. Alguns pesquisadores têm encontrado que o apoio afetivo e emocional do cônjuge prediz melhor a satisfação conjugal (Mickelson, Claffey, \& Williams, 2006).

As teorias biológicas postulam que homens e mulheres processam eventos nos seus relacionamentos em níveis cardiovascular, endócrino, imunológico, neurossensorial e neurofisiológico de formas diferentes. Os homens seriam mais sensíveis do que as mulheres a estressores agudos, mulheres apresentariam alterações fisiológicas mais fortes e duradouras para conflitos conjugais do que homens (Kiecolt-Glaser \& Newton, 2001). Outros propõem que homens e mulheres diferem nas áreas em que enfrentaram desafios adaptativos distintos nos seus cursos evolutivos (Simpson \& Gangestad, 2001).

Por outro lado, Jackson et al. (2014) realizaram uma meta-análise envolvendo 226 amostras e 101.110 pessoas. Os resultados mostraram diferenças de efeito muito pequeno, com as mulheres levemente menos satisfeitas do que os homens. As análises adicionais indicaram que não houve diferenças quando os níveis de satisfação conjugal de maridos e esposas foram comparados na mesma relação (dados diádicos). Para Kurdek (2005) é possível que essas diferenças sexuais entre homens e mulheres, com dados não relacionados, sejam devidas às amostras enviesadas.

No presente estudo, a condição um filho apresentou o menor nível de satisfação conjugal na comparação com as condições sem filhos e com apenas um filho em SIC e $\mathrm{ESC}_{\text {total }}$ Tam- 
bém foi encontrada diferença significativa entre as condições um filho e três ou mais filhos em SAOE. Isso poderia estar relacionado com a transição para a parentalidade, um período de adaptação dos cônjuges aos novos papéis de pai e profissional (Hernandez \& Hutz, 2009; Lawrence, Rothman, Cobb, \& Bradbury, 2010). Após a superação do impacto do primeiro filho, os filhos seguintes seriam absorvidos com mais facilidade, haja vista que não foram encontradas diferenças significativas entre o grupo sem filhos e os grupos com dois e três ou mais filhos. Em geral, a existência de filhos também pode acarretar restrições à liberdade dos cônjuges, especialmente quando estes exercem uma profissão e, muitas vezes, trabalham muito e em trabalho estressante (Twenge, Campbell, \& Foster, 2003).

Neste estudo, no mínimo, foram geradas evidências razoáveis de validade fatorial, validade de critério e fidedignidade para os escores da versão reduzida da ESC. Contudo, a amostra utilizada no estudo atual é de conveniência e limitada à região metropolitana do Rio de Janeiro, portanto, esses achados devem ser considerados nessa condição restrita em que foram produzidos. Por outro lado, esses participantes eram cidadãos comuns, quando é frequente o uso de amostras universitárias nesta área de pesquisa. Além disso, em média, os indivíduos examinados eram adultos de meia-idade e estavam em relações conjugais efetivas (casados ou em união estável) há longo tempo, condições importantes quando se investiga satisfação conjugal. Sugere-se futuras pesquisas com amostras maiores e mais abrangentes da população brasileira que possam acrescentar mais dados para a consolidação da validade estrutural da ESC.

\section{Referências}

Ahmadi, H., \& Sadeghi, M. (2016). The role of socio - Economic status in marital satisfaction. Turkish Journal of Psychology, 31(77), 43-47.

Arbuckle, (2009). Amos User's Guide. Chicago, IL: Statistical Product and Service Solutions.

Benetti, S. P. C. (2006). Conflito conjugal: Impacto no desenvolvimento psicológico da criança e do adolescente. Psicologia: Reflexão e Crítica, 19 ,
261-268. doi:http://dx.doi.org/10.1590/S010279722006000200012

Bentler, P. M. (1977). Factor Simplicity Index and Transformations. Psychometrika, 42(2), 277 295. doi:10.1007/BF02294054

Bolsoni-Silva, A. T., \& Marturano, E. M. (2010). Relacionamento conjugal, problemas de comportamento e habilidades sociais de pré-escolares. Psicologia: Teoria e Prática, 26(1), 67-75.

Buja, A., \& Eyuboglu, N. (1992). Remarks on parallel analysis. Multivariate Behavioral Research, 27(4), 509-540. doi:10.1207/ s15327906mbr2704 2

Byrne, B. (2010). Structural Equation Modeling with AMOS. Basic Concepts, Applications, and Programming. New York: Routledge Taylor \& Francis Group.

Cohen, J. (1992). A Power Primer. Psychological Bulletin, 112(1), 155-159. doi:http://dx.doi. org/10.1037/0033-2909.112.1.155

Conway, J. M., \& Huffcutt, A. (2003). A review and evaluation of exploratory factor analysis practices in organizational research. Organizational Research Methods, 6(2), 147-168. doi:10.1177/1094428103251541

Costello, A. B., \& Osborne, J. W. (2005). Best practices in Exploratory Factor Analysis: Four recommendations for getting the most from your analysis. Practical Assessment, Research \& Evaluation, 10(7). Retrieved from http://pareonline.net/getvn.asp? $v=10 \& n=7$

Damásio, B. F. (2012). Uso da análise fatorial exploratória em psicologia. Avaliação Psicológica, 11(2), 213-228.

Dela Coleta, M. F. (1989). A Medida da Satisfação Conjugal: Adaptação de uma Escala. Psico, 18(2), 90-112.

Dela Coleta, M. F. (1992). Locus de controle e satisfação conjugal. Psicologia: Teoria e Pesquisa, 8, 243-252.

Dominguez Lara, A. S. (2014). ¿ Matrices Policóricas/Tetracóricas o Matrices Pearson? Un estudio metodológico. Revista Argentina de Ciencias del Comportamiento, 6(1), 39-48.

Ferrando, P. J., \& Anguiano-Carrasco, C. (2010). El análisis factorial como técnica de investigación en psicología. Papeles del Psicólogo, 31(1), 18-33.

Gaskin, C. J., \& Happell, B. (2014). On exploratory factor analysis: A review of recent evidence, an 
assessment of current practice, and recommendations for future use. International Journal of Nursing Studies, 51, 511-521. doi:http://dx.doi. org/10.1016/j.ijnurstu.2013.10.005

Gottman, J. M., \& Silver, N. (2000). The Seven Principles for Making Marriage Work: A Practical Guide from the Country's Foremost Relationship Expert. New York: Three Rivers Press.

Hair, J. F., Black, W. C., Babin, B. J., Anderson, R. E., \& Tatham, R. L. (2009). Análise Multivariada de Dados. Porto Alegre, RS: Bookman.

Henson, R. K., \& Roberts, J. K. (2006). Use of exploratory factor analysis in published research. Common errors and some comment on improved practice. Educational and Psychological Measurement, 66(3), 393-416.

Hernandez, J. A. E., \& Hutz, C. S. (2009). Transição para a parentalidade: Ajustamento conjugal e emocional. Psico, 40(4), 414-421.

Izquierdo, I., Olea, J., \& Abad, F. J. (2014). Exploratory factor analysis in validation studies: Uses and recommendations. Psicothema, 26(3), 395400. doi:10.7334/psicothema2013.349

Jackson, J. B., Miller, R. B., Oka, M., \& Henry, R. G. (2014). Gender differences in Marital Satisfaction: A Meta-analysis. Journal of Marriage and Family, 76(1), 105-129. doi:10.1111/ jomf.12077

Kelley, T. L. (1935). Essential Traits of Mental Life, Harvard Studies in Education. Cambridge, MA: Harvard University Press.

Kiecolt-Glaser, J. K., \& Newton, T. L. (2001). Marriage and health: His and hers. Psychological Bulletin, 127(4), 472-503. doi:http://dx.doi. org/10.1037/0033-2909.127.4.472

Kurdek, L. A. (2005). Gender and Marital Satisfaction Early in Marriage: A Growth Curve Approach. Journal of Marriage and Family, 67(1), 68-84. doi:10.1111/j.00222445.2005.00006.x

Laros, J. A. (2005). O uso da análise fatorial: algumas diretrizes para pesquisadores. In L. Pasquali (Ed.), Análise fatorial para pesquisadores (pp. 163-184). Brasília, DF: Laboratório de Pesquisa em Avaliação e Medida.

Lawrence, E., Rothman, A. D., Cobb, R. J., \& Bradbury, T. N. (2010). Marital satisfaction across the transition to parenthood: Three eras of research. In M. S. Schulz, M. K. Pruett, P. K. Kerig, \& R. D. Parke (Eds.), Strengthening cou- ple relationships for optimal child development: Lessons from research and intervention (pp. 97114). Washington, DC: American Psychological Association.

Lorenzo-Seva, U. (2003). A factor simplicity index. Psychometrika, 68(1), 49-60. doi:10.1007/ BF02296652

Lorenzo-Seva, U., \& Ferrando, P. J. (2015). Manual of The Program FACTOR. Tarragona, Spain: Universitat Rovira i Virgili. Retrieved from http://psico.fcep.urv.es/utilitats/factor/index. html

Lorenzo-Seva, U., Timmerman, M. E., \& Kiers, H. A. L. (2011). The Hull method for selecting the number of common factors. Multivariate Behavioral Research, 46(2), 340-364. doi:10.1080/00 273171.2011 .564527

Lloret-Segura, S., Ferreres-Traver, A., Hernández-Baeza, A., \& Tomás-Marco, I. (2014). El análisis factorial exploratorio de los ítems: una guía práctica, revisada y actualizada. Anales de Psicología, 30(3), 1151-1169. doi:http://dx.doi. org/10.6018/analesps.30.3.199361

Machado, L. M. (2007). Satisfação e insatisfação no casamento: Os dois lados de uma mesma moeda? (Dissertação de mestrado, Programa de Pós-Graduação em Psicologia, Universidade Federal de Uberlândia, MG, Brasil).

Mardia, K. V. (1970). Measures of multivariate skewness and kurtosis with applications. Biometrika, 57(3), 519-530. doi:10.2307/2334770

Marôco, J. (2014). Análise de Equações Estruturais. Fundamentos Teóricos, Software \& Aplicações. Pêro Pinheiro, Portugal: Report Number.

Mickelson, K. D., Claffey, S. T., \& Williams, S. L. (2006). The moderating role of gender and gender role attitudes on the link between spousal support and marital quality. Sex Roles, 55(1), 73-82. doi:10.1007/s11199-006-9061-8

Mihalcea, A., Iliescu, D., \& Dincă, M. (2013). The Influence of the Educational Level on Couple Relationships. Procedia - Social and Behavioral Sciences, 78, 41-45. doi:10.1016/j. sbspro.2013.04.247

Minnotte, K. L., Minnotte, M. C., \& Pedersen, D. E. (2013). Marital satisfaction among dual-earner couples: Gender ideologies and family-to-work conflict. Family Relations, 62(4), 686-698. doi:10.1111/fare.12021 
Mirecki, R. M., Chou, J. L., Elliott, M., \& Schneider, C. M. (2013). What factors influence marital satisfaction? Differences between first and second marriages. Journal of Divorce \& Remarriage, 54(1), 78-93. doi:http://dx.doi.org/10.1080/105 02556.2012 .743831

Norgren, M. B. P., Souza, R. M., Kaslow, F., Hammerschmidt, H., \& Sharlin, S. A. (2004). Satisfação conjugal em casamentos de longa duração: Uma construção possível. Estudos de Psicologia (Natal), 9(3), 575-584. doi:http://dx.doi. org/10.1590/S1413-294X2004000300020

Oliveira, M. G. S., Falcone, E. M. de O., \& Ribas, R. C., Jr. (2009). A avaliação das relações entre a empatia e a satisfação conjugal: Um estudo preliminar. Interação em Psicologia, 13(2), 287-298. doi:http://dx.doi.org/10.5380/psi.v13i2.8025

Pick de Weiss, S., \& Andrade Palos, P. (1988a). Desarrolllo y validacion de la Escala de Satisfaccion Marital. Psiquiatria, 1, 9-20.

Pick de Weiss, S., \& Andrade Palos, P. (1988b). Diferencias sociodemográficas en la satisfacción marital: el caso de México. Revista de Psicología Social, 3, 91-97.

Pick de Weiss, S., \& Andrade Palos, P. (1988c). Relación entre el número de hijos, la satisfacción marital y la comunicación con el cónyuge. Salud Mental, 11, 15-18.

Ribeiro, C. M., Pinho, V. D., \& Falcone, E. M. O. (2011). A influência da raiva e da empatia sobre a satisfação conjugal. Aletheia, 35-36, 7-21.

Rostami, A., Ghazinour, M., Nygren, L., \& Richter, J. (2014). Marital satisfaction with a special focus on gender differences in medical staff in Tehran, Iran. Journal of Family Issues, 35(14), 19401958. doi:10.1177/0192513X13483292

Sardinha, A., Falcone, E. M. O., \& Ferreira, M. C. (2009). As relações entre a satisfação conjugal e as habilidades sociais percebidas no cônjuge. Psicologia: Teoria e Pesquisa, 25(3), 329-336. doi:http://dx.doi.org/10.1590/S010237722009000300013

Schmid, J., \& Leiman, J. N. (1957). The development of hierarchical factor solutions. Psychometrika, 22, 53-61. doi:10.1007/BF02289209

Sbicigo, J. B., \& Lisbôa, C. S. de M. (2009). Habilidades sociais e satisfação conjugal: Um estudo correlacional. Revista Brasileira de Terapias Cognitivas, 5, 73-81.
Scorsolini-Comin, F., \& Santos, M. A. (2011a). Relações entre bem-estar subjetivo e satisfação conjugal na abordagem da psicologia positiva. Psicologia: Reflexão e Crítica, 24, 658-665. doi:http://dx.doi.org/10.1590/S010279722011000400005

Scorsolini-Comin, F. \& Santos, M. A. (2011b). Ajustamento diádico e satisfação conjugal: Correlações entre os domínios de duas escalas de avaliação da conjugalidade. Psicologia: Reflexão e Crítica, 24(3), 439-447. doi:http://dx.doi. org/10.1590/S0102-79722011000300007

Scorsolini-Comin, F., \& Santos, M. A. (2012). A medida positiva dos afetos: Bem-estar subjetivo (BES) em pessoas casadas. Psicologia: Reflexão e Crítica, 25, 11-20. doi:http://dx.doi. org/10.1590/S0102-79722012000100003

Simpson, J. A., \& Gangestad, S. W. (2001). Evolution and relationships: A call for integration. Personal Relationships, 8(4), 341-355. doi:10.1111/j.1475-6811.2001.tb00044.x

Solomon, B. C., \& Jackson, J. J. (2014). Why do personality traits predict divorce? Multiple pathways through satisfaction. Journal of Personality and Social Psychology, 106(6), 978-996. doi:http://dx.doi.org/10.1037/a0036190

Timmerman, M. E., \& Lorenzo-Seva, U. (2011). Dimensionality assessment of ordered polytomous items with parallel analysis. Psychological Methods, 16(2), 209-220. doi:10.1037/a0023353

Twenge, J., Campbell, K., \& Foster, C. (2003). Parenthood and marital satisfaction: A metaanalytic review. Journal of Marriage and Family, 65(3), 574-583. doi:10.1111/j.17413737.2003.00574.x

Villa, M. B., \& Del Prette, Z. A. P. (2013). Marital satisfaction: The role of social skills of husbands and wives. Paidéia (Ribeirão Preto), 23(56), 379-388. doi:http://dx.doi.org/10.1590/198243272356201312

Wolff, H.-G., \& Preising, K. (2005). Exploring item and higher order factor structure with the Schmid-Leiman solution: Syntax codes for SPSS and SAS. Behavior Research Methods, 37, 48-58. doi:10.3758/BF03206397

Recebido: 16/05/2016

$1^{a}$ revisão: $17 / 12 / 2016$

Aceite final: 28/12/2016 